\title{
Competitive Adsorption of a Multi-functional Amine and Phenol Surfactant with Ethanol on Hematite from Non- Aqueous Solution
}

DOI:

10.1021/acs.jpcb.8b09704

\section{Document Version}

Accepted author manuscript

Link to publication record in Manchester Research Explorer

Citation for published version (APA):

Chia, C. L., Alloway, R. M., Jephson, I., Clarke, S. M., Filip, S. V., Siperstein, F., \& Avendano, C. (2019). Competitive Adsorption of a Multi-functional Amine and Phenol Surfactant with Ethanol on Hematite from NonAqueous Solution. The Journal of Physical Chemistry Part B, 123(6), 1375-1383. https://doi.org/10.1021/acs.jpcb.8b09704

\section{Published in:}

The Journal of Physical Chemistry Part B

\section{Citing this paper}

Please note that where the full-text provided on Manchester Research Explorer is the Author Accepted Manuscript or Proof version this may differ from the final Published version. If citing, it is advised that you check and use the publisher's definitive version.

\section{General rights}

Copyright and moral rights for the publications made accessible in the Research Explorer are retained by the authors and/or other copyright owners and it is a condition of accessing publications that users recognise and abide by the legal requirements associated with these rights.

\section{Takedown policy}

If you believe that this document breaches copyright please refer to the University of Manchester's Takedown Procedures [http://man.ac.uk/04Y6Bo] or contact uml.scholarlycommunications@manchester.ac.uk providing relevant details, so we can investigate your claim.

\section{OPEN ACCESS}


This document is confidential and is proprietary to the American Chemical Society and its authors. Do not copy or disclose without written permission. If you have received this item in error, notify the sender and delete all copies.

\section{Competitive Adsorption of a Multi-functional Amine and Phenol Surfactant with Ethanol on Hematite from Non- aqueous Solution}

\begin{tabular}{|r|l|}
\hline Journal: & The Journal of Physical Chemistry \\
\hline Manuscript ID & jp-2018-09704e.R2 \\
\hline Manuscript Type: & Article \\
\hline Author: & n/a \\
\hline Complete List of Authors: & $\begin{array}{l}\text { Chia, Chung Lim; University of Manchester, School of Chemical } \\
\text { Engineering and Analytical Science } \\
\text { Alloway, Richard; University of Cambridge Department of Chemistry, } \\
\text { Chemistry } \\
\text { Jephson, Izaak; University of Cambridge Department of Chemistry } \\
\text { CLARKE, Stuart M.; BP Institute and Department of Chemistry, } \\
\text { University of Cambridge, Cambridge CB3 OEZ, U.K., BP Institute and } \\
\text { Department of Chemistry } \\
\text { Filip, Sorin; BP, Formulated Products Technology } \\
\text { Siperstein, Flor; University of Manchester, School of Chemical } \\
\text { Engineering } \\
\text { Avendano, Carlos; University of Manchester, School of Chemical } \\
\text { Engineering and Analytical Science }\end{array}$ \\
\hline
\end{tabular}

\section{SCHOLARONE Manuscripts}




\title{
Competitive Adsorption of a Multi-functional Amine and Phenol Surfactant with Ethanol on Hematite from Non-Aqueous Solution
}

\author{
Chung-Lim Chia, ${ }^{\dagger}$ Richard M. Alloway, ${ }^{\ddagger}$ Izaak Jephson, ${ }^{\ddagger}$ Stuart M. Clarke, ${ }^{\ddagger}$ \\ Sorin V. Filip, "Flor R. Siperstein, ${ }^{\dagger}$ and Carlos Avendaño, ${ }^{\dagger}$ \\ $\dagger$ School of Chemical Engineering and Analytical Science, The University of Manchester, \\ Oxford Road, Manchester, M13 9PL, U.K. \\ $\ddagger$ Department of Chemistry and BP Institute, Cambridge University, Cambridge, CB2 \\ $1 E W, U . K$. \\ ФBP Formulated Products Technology, Research and Innovation, Technology Centre, \\ Whitchurch Hill, Pangbourne, Berkshire, RG8 7QR, U.K. \\ E-mail: carlos.avendano@manchester.ac.uk
}




\begin{abstract}
Surfactants, which contain phenol and amine groups, are commonly used in industry to protect metallic surfaces and their efficiency depends strongly on factors such as pressure and temperature, solvent properties, and the presence of other surfactants in the system. In this work, we present a molecular simulation study of the competitive adsorption between a multi-functional phenol and amine surfactant model and ethanol at the oil/solid interface formed between iso-octane and a model hematite $\left(\alpha-\mathrm{Fe}_{2} \mathrm{O}_{3}\right)$ slab. We show that the surfactant strongly adsorbs at the iso-octane/hematite interface in the absence of ethanol at moderate temperatures. As the concentration of ethanol is increased, the ethanol molecules compete effectively for the adsorption sites on the iron oxide surface. This competition drives the surfactant molecules to remain in the bulk-solution while ethanol forms an ordered and strongly coordinated layers at the oil/solid interface, despite the well-known complete miscibility of ethanol in iso-octane in bulk at standard conditions. Potential of mean force calculations show that the free energy of adsorption of the surfactant is approximately two times larger than for a single ethanol molecule, but the simulations also reveal that a single surfactant chain needs to displace up to five ethanol molecules to adsorb onto the surface. The end result is a more favorable ethanol adsorption which agrees with the experimental analysis of similar oil/iron oxide systems also reported in this work.
\end{abstract}

\title{
Introduction
}

Adsorption of organic molecules such as ligands and surfactants remains one of the most common methods to protect and modify the properties of solid surfaces. It has important industrial implications in areas such as sensing and molecular recognition, selective crystal growth, heterogeneous catalysis, tribology, and corrosion protection. ${ }^{1}$ In the automotive industry, for example, a variety of surfactants are used in fuel formulation to protect and lubricate the car engine surfaces. ${ }^{2,3}$ The adsorption of surfactants is a complex phenomenon 

face chemistry of the solid substrate, solid defects and surface morphology due mechanical stresses. Some of these factors have been studied closely using state-of-the-art experimental techniques. ${ }^{4-11}$ On top of these studies, molecular simulation methods have also been important in understanding the adsorption phenomena of surfactants as they offer atomistic insights about the mechanisms involved in the process. ${ }^{12-14}$ To date, increasing numbers of computational studies have been performed to investigate complex phenomena taking place at liquid/solid interfaces such as mechanisms of adsorption, ${ }^{15}$ and surfactant self-assembly as a function of concentration, ${ }^{16-18}$ chain length, ${ }^{19}$ aqueous salt concentration, ${ }^{20-22}$ surface morphology ${ }^{23-28}$ and interaction parameters. ${ }^{29,30}$

Most of the computer simulation studies of adsorption of surfactants consider aqueous systems. However, in many industrial applications, adsorption of surfactants takes place at oil/solid interfaces, which is a phenomenon not well understood yet. Some recent simulation studies have focused on the orientation and lubrication properties of surfactants in non-polar phases on mica and iron oxide surfaces where, for example, it has been observed that hexadecylamine forms a monolayer on iron oxide at high surfactant concentration with the hydrophilic groups attached to the surface and their hydrophobic tails exposed to the oil phase. ${ }^{31}$ Similar phenomenon has been observed in systems formed by fatty acids. ${ }^{32}$ These observations are in accordance with some of the experimental studies in the literature. ${ }^{5,6}$ The situation becomes more challenging to understand in the case of high-performance formulations. These systems usually contain a variety of surfactants, of which many have complex molecular structures. The performance of these high-performance formulations can also be affected by other molecules present in the system. For example, ethanol has been added to gasoline blends to enhance the octane number and reduce environmental impact of the fuel since the 1980s. ${ }^{33-36}$ However, the polar group of ethanol may compete with other surfactants for active sites on the surface thus reducing their protective efficiency. Therefore, the impact that ethanol has on active surfaces should not be neglected. Indeed, experimental evidence 


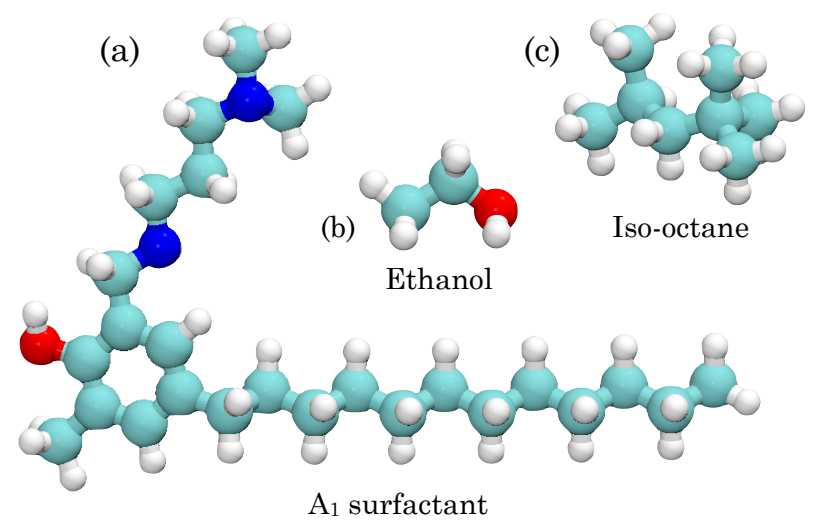

Figure 1: Molecular structure of the systems comprising the oil phase in this study: (a) Mannich base surfactant, (b) ethanol, and (c) iso-octane.

obtained in this work suggests that adsorption of certain surfactants can be suppressed by the presence of ethanol.

The goal of this study is to understand the competitive adsorption between ethanol and a model of commonly used surfactant, which is typically present in gasoline blends, at the oil/solid interface formed between iso-octane and hematite using molecular dynamics simulations.

\section{Simulation Methods}

We use molecular-dynamics (MD) simulation to gain a microscopic understanding of the adsorption of a Mannich base (amine) surfactant model at the oil/solid interface formed by iso-octane and a slab of hematite $\left(\alpha-\mathrm{Fe}_{2} \mathrm{O}_{3}\right)$. The surfactant model is typically synthesised by Mannich base reactions between amines and aldehydes, and for this particular study we consider the 2-[ (3-(dimethylamino) propyl)amino] methyl-4-dodecyl-6-methylphenol ${ }^{37-39}$ surfactant that contains a polar head group with phenol and amino groups, and a tail group comprising of an aliphatic $\mathrm{C}_{12}$ alkyl chain. The molecular structure of this model surfactant is shown in Figure 1(a), and for simplicity hereafter we will refer to this molecule as simply $\mathrm{A}_{1}$. 
Iso-octane is used to represent the oil phase while a iron-terminated hematite slab with the (0001) crystallographic plane exposed to the fluid phase represent our model surface. Under ambient conditions, hematite is the most common form of iron oxide on steel surfaces. ${ }^{40,41}$ The iron-terminated surface is used as it has the pristine hematite structure with lowest surface energy based on ab initio calculations. ${ }^{42}$ Our simulation setup is constructed as follow. First, the structure of iron-terminated hematite (0001) surface is built from crystallographic information $^{43}$ and replicated by $16 \times 16 \times 2$ unit cells $(80.6 \AA \times 69.8 \AA \times 27.5 \AA)$ along the three Cartesian coordinates. The force field used to model the hematite slab was taken from the model of Berro et al. ${ }^{44}$ which has previously been used to model the adsorption of surfactants on iron oxides. ${ }^{31,45}$ The interactions between iron-iron, iron-oxygen and oxygenoxygen within the solid structure are not considered since the slab is described as rigid in this work. The OPLS-AA force field parameterization of Price et al. ${ }^{46}$ is used to represent the molecular interactions between iso-octane, ethanol, and surfactant $A_{1}$, and the parameters are given in the supplementary information. Standard Lorentz-Berthelot mixing rules are used to represent the cross interactions. The OPLS-AA force field has previously been used to model mixture of alkane-alcohol, alkane-amine and alcohol-amine with satisfactory results for various thermodynamic properties. ${ }^{47}$ Bulk liquid simulations have also been performed in this work to ensure that the OPLS-AA force field represents well the miscibility of ethanol and iso-octane at various temperatures in the range of 270-390 K. We observed that the OPLS-AA force field parameterization used in this work reproduces the bulk experimental density of an equimolar mixture of iso-octane and ethanol (less than $2 \%$ deviation), ${ }^{48}$ as well as the full miscibility of both components. All LJ interactions have a cutoff of $10 \AA$ and electrostatic interactions are handled using the PPPM method. All simulations have been performed using LAMMPS. ${ }^{49}$

The hematite slab is located in the centre of the simulation box with the two (0001) crystallographic planes oriented perpendicular to the $z$ direction and exposed to the fluid phase. The different molecules in the fluid phase are inserted in the simulation box using the 
Packmol suite. ${ }^{50}$ The systems are equilibrated to a pressure of 1 bar and to the appropriate temperatures using MD simulations in the isobaric-isothermal NPT ensemble, allowing only the box length in $z$ direction to vary. More formally, the simulations take place in the $N P_{N} A T$ ensemble, where the normal pressure $P_{N}=P_{z z}$ and the transverse area $A=L_{x} L_{y}$ are kept constant. Depending on the mixture composition, the resulting box length in the $z$ direction ranges from 110 to $120 \AA$. After equilibration, MD simulations are performed using the canonical $N V T$ ensemble to study the adsorption mechanism of the fluid molecules on the solid substrate. Newton's second law is integrated using the velocity Verlet algorithm using a 1 fs time step and periodic boundary conditions in all three Cartesian coordinates for all simulations so that the solid slab becomes infinite in the $x y$ plane. Temperature and pressure are controlled employing Nosé-Hoover dynamics. ${ }^{51,52}$ Details on the number of molecules used for each species are summarised in Table 1.

We have also performed MD simulations using umbrella sampling to study, in more detail, the adsorption of $\mathrm{A}_{1}$ at the oil/hematite interface with and without the presence of ethanol in the system. Additionally, umbrella sampling simulations of ethanol with and without the presence of $A_{1}$ are also used to compare the adsorption affinities between the two molecular species. MD simulations using umbrella sampling allow one exploring more efficiently all possible configurations that are not energetically favourable by applying a bias harmonic potential to pull the molecules of interest to the solid substrate. The resulting trajectories are used to calculate the potential mean force (PMF) using the Weighted Histogram Analysis method (WHAM) ${ }^{53}$ along the reaction coordinate. For the umbrella sampling simulation for $\mathrm{A}_{1}$, the reaction coordinate is taken as distance along the $z$ direction between the centre of the aromatic ring of the phenol group and the outermost surface atoms of hematite, while for ethanol the reaction coordinate is taken with respect to the oxygen atom. A window size of $0.5 \AA$ is used to ensure that the histograms overlap each other. For each window along the reaction coordinate, a simulation of $5 \mathrm{~ns}$ is performed in the $N V T$ ensemble. The free energy reference value is taken as zero for distances larger than $25.0 \AA$ which is considered 
to be a distance far enough from the surface. Finally, the adsorption free energy is taken as the global minimum value of the PMF.

The different systems analysed using MD simulations with and without umbrella sampling are described in Table 1. For simulations using MD with umbrella sampling (MD-US), a system containing a single $\mathrm{A}_{1}$ surfactant in a solvent containing 1680 molecules of iso-octane at $293 \mathrm{~K}$ is used, in which a bias harmonic potential is applied to the center of the aromatic ring of the phenol group to pull the molecule of $\mathrm{A}_{1}$ towards the surface. The bias harmonic potential is computed based on a force constant of $7.0 \mathrm{kcal} \mathrm{mol}^{-1} \AA^{-1}$ and the equilibrium distance is sequentially decreased from $25.0 \AA$ to $0.0 \AA$. The resulting trajectories are used to calculate the PMF profiles of $\mathrm{A}_{1}$ on hematite. A similar calculation is performed for a single ethanol molecule in iso-octane solvent and the bias potential is applied to the oxygen atom. Next, the calculations for the PMF profile of a single $A_{1}$ surfactant are repeated but now including different concentrations of ethanol in the system corresponding to $0,64,128$, 256, 512, and 768 molecules at $293 \mathrm{~K}$. These calculations aim to understand the effects of ethanol concentration on the free energy of adsorption of $\mathrm{A}_{1}$. The effect of temperature on the PMF profile of a single molecule of $\mathrm{A}_{1}$ is also analysed using a system containing 768 ethanol molecules at temperatures corresponding to $293 \mathrm{~K}, 313 \mathrm{~K}, 333 \mathrm{~K}, 353 \mathrm{~K}$, and 373 K.

For the unbiased MD simulations, systems containing 1680 molecules of iso-octane and different concentrations of ethanol are considered. The results are used to analyse structural properties of the adsorbed ethanol layer at the oil/hematite interface. The number of ethanol molecules used for these calculations are 256, 512, and 768. Finally, the simulations are repeated by adding 16 molecules of $A_{1}$ to analyse the competitive adsorption between $A_{1}$ and ethanol on hematite. 
Table 1: Summary of the number of molecules used for MD simulations without and with using umbrella sampling (MD-US) to calculate the PMFs.

\begin{tabular}{lllll}
\hline Iso-octane & Ethanol & $\mathrm{A}_{1}$ & Temperature(s) / K & Simulation technique \\
\hline 1680 & - & 1 & 293 & MD-US \\
1680 & 1 & - & 293 & MD-US \\
1680 & $0,64,128,256,512,768$ & 1 & $293,313,333,353,373$ & MD-US \\
1680 & $256,512,768$ & - & 293 & MD \\
1680 & $256,512,768$ & 16 & 293 & MD \\
\hline
\end{tabular}

\section{Experimental Method}

To further confirm the competitive adsorption of $\mathrm{A}_{1}$ and ethanol, adsorption isotherms of mixtures of ethanol, $\mathrm{A}_{1}$, and iso-octane on hematite are determined by the solution depletion method. All experiments are performed at room temperature and they follow the same methodology previously used in reference 5. These experiments essentially measure the fall in solution concentration of a surfactant in contact with a powdered substrate to estimate the amount adsorbed. The changes in concentration are measured using UV-Vis spectroscopy for the case of $\mathrm{A}_{1}$, and quantitative ${ }^{1} \mathrm{H}$ NMR for the case of ethanol. The substrate was powdered $\mathrm{Fe}_{2} \mathrm{O}_{3}$ from Sigma, with a specific surface area determined by nitrogen BET of $5.12 \mathrm{~m}^{2} \mathrm{~g}^{-1}$.

\section{Results and Discussions}

\section{Potentials of mean force for surfactant $A_{1}$ and ethanol}

We analyse the free energy required to bring either a single molecule of $\mathrm{A}_{1}$ or a single molecule of ethanol from infinity towards the oil/hematite interface as a first step towards understanding the competitive adsorption of $\mathrm{A}_{1}$ and ethanol on hematite. These free energies obtained from the calculations of the PMF using umbrella sampling simulations provide information of the affinity of $\mathrm{A}_{1}$ and ethanol to adsorb to the solid substrate without, in the first instance, considering interference between each other. The results for the PMF profiles 


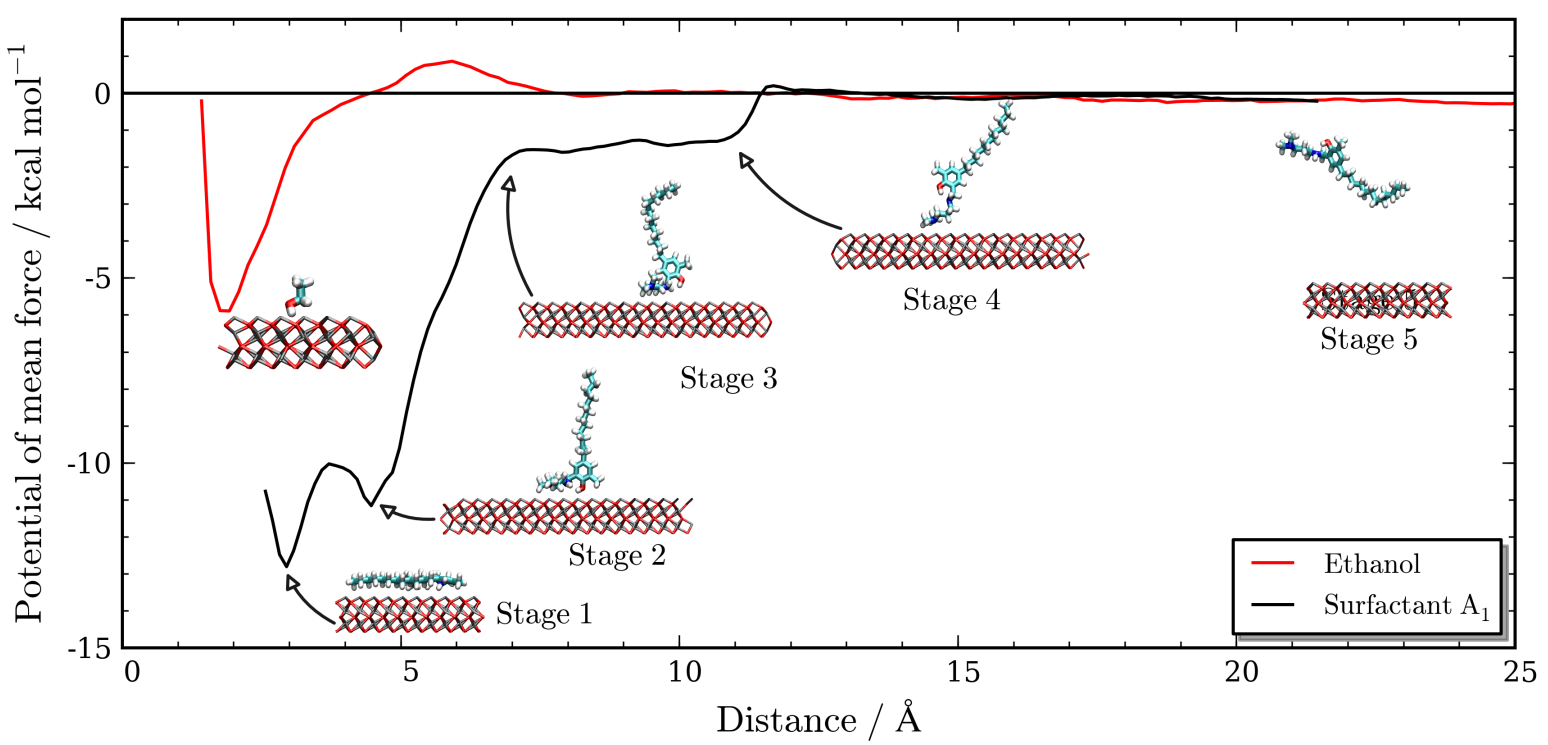

Figure 2: Potential of mean force of a single ethanol molecule and single surfactant $\mathrm{A}_{1}$ on hematite (0001) surface. In the profile for $A_{1}$, snapshots of 5 adsorption stages are also presented, while for ethanol a single stage is shown. The origin $z=0$ corresponds to the outermost solid atom position.

are presented in Figure 2, where it is clearly observed that the PMF of surfactant $A_{1}$ has two pronounced minima, one at a distance of $2.94 \AA$ of magnitude $-12.9 \mathrm{kcal} \mathrm{mol}^{-1}$, and a second at a distance of $4.46 \AA$ of magnitude $-11.1 \mathrm{kcal} \mathrm{mol}^{-1}$. Both peaks observed in the PMF profile of $\mathrm{A}_{1}$ are clearly more pronounced than the single minimum observed for an ethanol molecule located at a distance of $1.91 \AA$ of magnitude $-5.8 \mathrm{kcal} \mathrm{mol}^{-1}$, indicating stronger adsorption affinity of $A_{1}$ on hematite. $A_{1}$ is significantly larger and has more polar functional groups than ethanol thus increasing the dispersion forces and electrostatic interactions with the surface. Therefore, it is not surprising to observe that $\mathrm{A}_{1}$ exhibits a stronger adsorption affinity compared to ethanol. The PMF profile also reveals that the surfactant has 5 different adsorption conformations (stages) represented by the minima and different turning points in the profile. Stage 1 corresponds to the adsorption of $\mathrm{A}_{1}$ adopting a flat conformation on the surface with the head and tail groups occupying the surface sites. Adsorption stages 2-4 take place when the polar amino and phenol groups are in contact with the surface and the aliphatic tail extends into the oil phase. Finally, stage 5 represents the complete desorption of $A_{1}$. The PMF profile of ethanol is completely different to the one of $A_{1}$, exhibiting only 
(a)

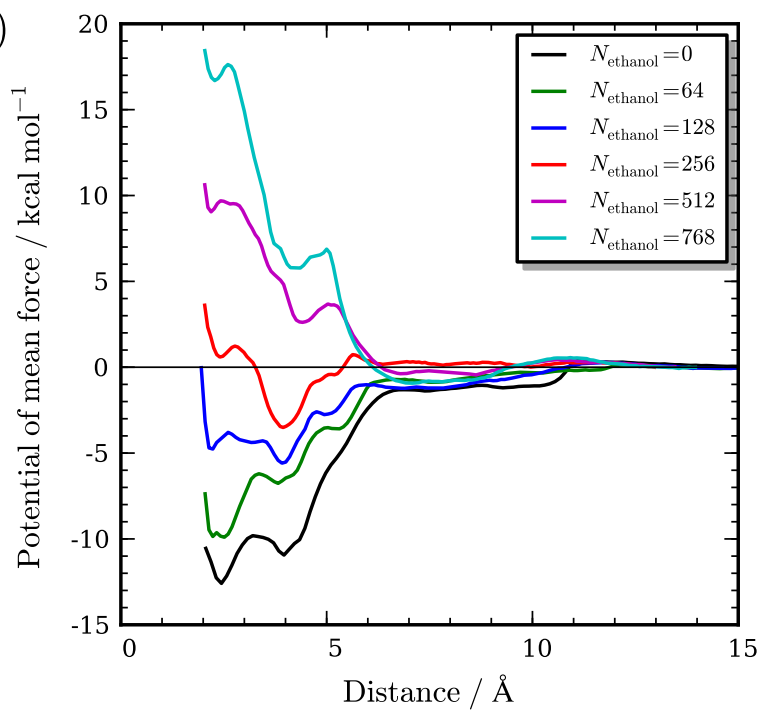

(b)

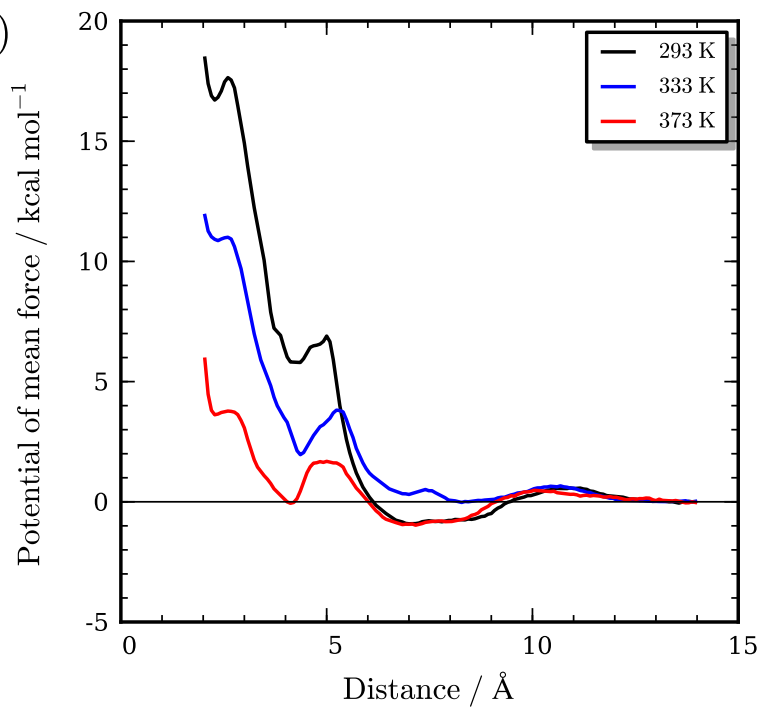

Figure 3: (a) PMF profile of surfactant $\mathrm{A}_{1}$ for six different numbers of ethanol molecules at $293 \mathrm{~K}$. $N_{\text {ethanol }}$ denotes the number of ethanol molecules. (b) PMF profile of surfactant $\mathrm{A}_{1}$ at three different temperatures for a system containing 768 ethanol molecules.

one adsorption conformation represented by a single minimum in PMF that is characterised by the interaction of the $\mathrm{OH}$ group with the surface and the alkyl group exposed to the oil phase.

From the previous results is evident that the affinity of $A_{1}$ to the hematite surface is stronger than that of ethanol. To assess whether these affinities are affected when the two 
Figure 4: Values of the PMF of $A_{1}$ reported in Figure ??fig:difftemp(a) as a function of the number of ethanol molecules evaluated at the first (blue circles) and second (red squares) minima. Solid lines represent best fits.

species compete between each other for the adsorption sites, we have carried out calculations of the PMF of $\mathrm{A}_{1}$ at different ethanol concentrations using umbrella sampling simulations at $293 \mathrm{~K}$. The number of molecules of ethanol analysed, $N_{\text {ethanol }}$, are indicated in Table 1 and the results are shown in Figure 3(a). Regardless of the ethanol concentration studied, the two minima in the PMF originally, at distances of $\sim 2.94 \AA$ and $\sim 4.46 \AA$, remain almost at the same positions, but their corresponding magnitudes are shifted towards higher values as the number of ethanol molecules is increased until the adsorption of $A_{1}$ on hemitite becomes completely unfavourable, i.e., until the PMF profile becomes positive for all distances. This is a counter-intuitive observation considering the strong affinity of $A_{1}$ to hematite. Moreover, it is also clear that as the number of ethanol molecules is increased beyond $N_{\text {ethanol }}=128$, the second minimum in the PMF becomes the global minimum suggesting configurations corresponding to the conformation of Stage 2 becoming the most stable ones. This change of conformation seems to be driven by the quick formation of an ethanol monolayer onto hematite that displaces the hydrophobic surfactant's alkyl chain towards the oil phase. The 
first and second minima observed in the PMF, with magnitudes $\Delta \mathrm{A}_{1^{\text {st }}}$ and $\Delta \mathrm{A}_{2^{\text {nd }}}$, represent the adsorption of the hydrophobic and hydrophilic groups respectively. The dependence of $\Delta \mathrm{A}_{1^{\text {st }}}$ and $\Delta \mathrm{A}_{2^{\text {nd }}}$ on the number of ethanol molecules is presented in Figure 4. Interpolation of $\Delta \mathrm{A}_{1 \text { st }}$ to zero, corresponding to a number of ethanol molecules of $N_{\text {ethanol }} \approx 290$, is the point at which the first minima becomes positive indicating destabilisation of the hydrophobic group, while the Interpolation of $\Delta \mathrm{A}_{2^{\text {nd }}}$ to zero, corresponding to $N_{\text {ethanol }} \approx 440$, is the point at which the second minima becomes positive indicating destabilisation of the hydrophilic group. When the surface is covered with ethanol, for a single $\mathrm{A}_{1}$ molecule to adsorb onto the surface requires the displacement of more than one ethanol molecule in order to accommodate its polar head group comprising three amino groups and the large phenol group. The number of ethanol molecules displaced per $A_{1}$ molecule can be estimated from the change in the amplitude of the first peak of the ethanol density profile, located at the oil/solid interface, before and after $\mathrm{A}_{1}$ is adsorbed. The analysis reveals that approximately 5 ethanol molecules need to be displaced in order for the head group to adsorb on the surface and adopting a conformation corresponding to Stage 2. In this case, the significant energy barrier of displacing a cluster of ethanol molecules is the key that prevents the adsorption of surfactant. This strong energy barrier is attributed to the strong order and hydrogen bond-network observed in the first ethanol layer.

Besides the strong effect that the concentration of ethanol has on the adsorption of $A_{1}$ on hematite, the effect of temperature needs to be analysed since high-performance surfactants are likely to be used at high temperature conditions. The results for the PMF profile for $\mathrm{A}_{1}$ at different temperatures and for a fixed number of $N_{\text {ethanol }}=768$ molecules are presented in Figure 3(b). The temperatures analysed correspond to $293 \mathrm{~K}, 333 \mathrm{~K}$, and $373 \mathrm{~K}$, which cover the temperature range from room temperature up to phase change temperature (boiling point) of iso-octane. ${ }^{54}$ Unlike in the case of increasing ethanol concentration, increasing the temperature of the system to $373 \mathrm{~K}$ shifts the PMF profile to lower values thus the adsoprtion of the surfactant become more favourable. This suggests that elevated temperature $\mathrm{A}_{1}$ is 

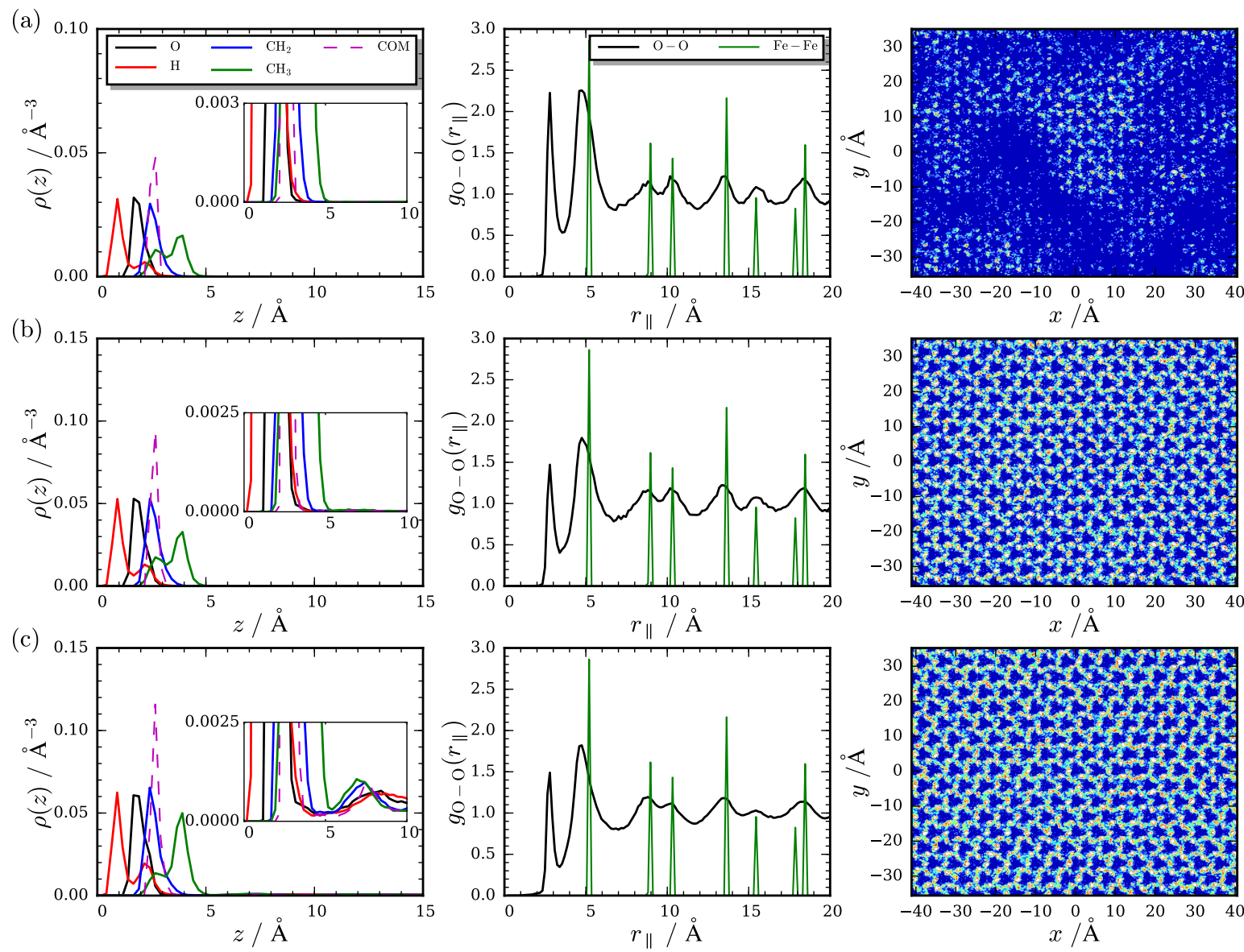

Figure 5: Density profile $\rho(z)$ of ethanol along the $z$ direction, two-dimensional oxygenoxygen radial distribution function $g_{\mathrm{O}-\mathrm{O}}\left(r_{\|}\right)$in the plane parallel to $x-y$ directions calculated for the first adsorbed ethanol layer, and two-dimensional density map of ethanol on hematite (0001) surface. The total number of ethanol molecules $N_{\text {ethanol }}$ in the three systems correspond to (a) 256, (b) 512 and (c) 768 molecules, respectively. The origin $z=0$ for the density profile corresponds to the position of the outermost solid atoms of the hematite slab. COM denotes the center of mass of ethanol.

able to compete with ethanol molecules for the adsorption sites of the solid substrate. This enhanced competition is attributed to the weakening of the hydrogen bonds in the first ethanol layer that reduces the order of the ethanol network and favours the adsorption of $\mathrm{A}_{1}$. 


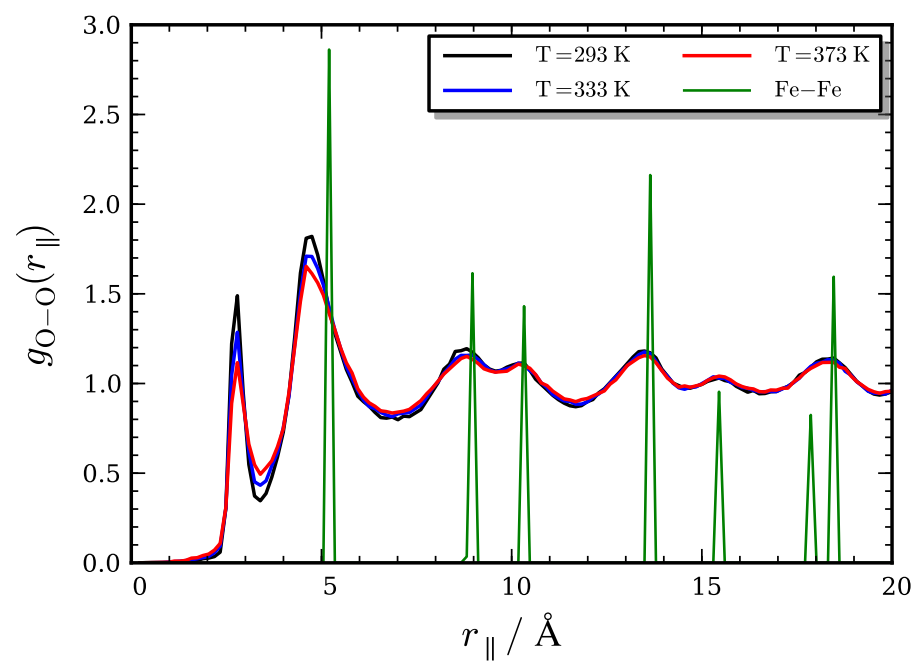

Figure 6: Two-dimensional oxygen-oxygen radial distribution functions $g_{\mathrm{O}-\mathrm{O}}\left(r_{\|}\right)$calculated for the first ethanol layers formed at temperatures corresponding to $293 \mathrm{~K}$ (black), $333 \mathrm{~K}$ (blue), $373 \mathrm{~K}$ (red). The number of ethanol molecules used is 768 . The iron-iron $g_{\mathrm{Fe}-\mathrm{Fe}}\left(r_{\|}\right)$ (green) for the iron atoms in the outmost solid layer of hematite is also included for comparison.

\section{Adsorption of ethanol and $A_{1}$ on the hematite}

We proceed now to discuss the results obtained from the molecular dynamics simulations for ethanol and $A_{1}$. We present first the results for the adsorption of ethanol at the oil/hematite interface, without considering the presence of $\mathrm{A}_{1}$, to study the structural properties of the strong layers of ethanol formed on the solid substrate. The results for the $50 \mathrm{~ns}$ long MD simulations using 256, 512 and 768 ethanol molecules in iso-octane are shown in Figure 5. The characterisation of the ethanol layers is carried out using a methodology similar to the one reported by Phan et al. ${ }^{55}$ First, the density profile $\rho(z)$ along the $z$ direction perpendicular to the solid surface provides evidence of the formation of adsorbed layers of ethanol on hematite. The results for the density profiles for each ethanol concentration are shown in Figure 5. The intense (first) peaks in the density profiles, particularly for the one with respect to the centre of mass of ethanol, indicate that all ethanol molecules migrate to the interface formed between iso-octane and hematite, forming a stable adsorbed layer, de- 
spite the fact that ethanol is completely miscible in iso-octane at room temperature in bulk conditions. The positions of the first peaks of hydrogen, oxygen, ethyl and methyl are 0.93, 1.93, 2.42 and $3.91 \AA$, respectively, and they remain at the same positions for all ethanol concentrations. Hydrogen is the closest atom to the surface followed by oxygen, ethyl group and methyl group. The results also confirm that the methyl and ethyl groups of ethanol are oriented away from the surface and into the oil phase whereas the $\mathrm{OH}$ group of ethanol is bound to the surface. As the concentration of ethanol is increased, the density of the first adsorbed layer, obtained from the intensity of the peak in the center of mass (COM) $\rho(z)$, increases from 0.05 to 0.10 to 0.12 molecules per $\AA^{3}$. The intensity of this first peak appears to reach a limiting value as all exposed iron sites of the (0001) surface of hematite become occupied. For all three cases, the average ethanol densities of the first layer are calculated to be $0.48,0.85$, and $1.09 \mathrm{~g} \mathrm{~cm}^{-3}$, respectively. Second adsorption layers are not observed in the case of 256 and 512 ethanol molecules since the concentration of ethanol is only enough to form a single layer. When the number of ethanol molecules is increased to 768, however, the density profile shown in Figure 5(c) indicates the formation of a partial second adsorbed layer. However, this second adsorbed layer is less defined than the first one as inferred from the weaker and broader second peaks at a distance of $7.63 \AA$ shown in the inset figure. These results agree qualitatively with the experimental results shown in Figure 7, where the adsorption isotherm indicates a rise in the amount of ethanol adsorbed as the concentration is increased, but the expected plateau at high concentration was not observed. The amount of ethanol adsorbed is also shown to be much more than expected from a single monolayer of ethanol at the oil/solid interface.

The in-plane oxygen-oxygen radial distribution function $g_{\mathrm{O}-\mathrm{O}}\left(r_{\|}\right)$, where $r_{\|}$is the radial distance between two ethanol molecules in the $x-y$ plane, calculated for the first adsorbed layer of ethanol describes the packing of the molecules in the layer. The results for $g_{\mathrm{O}-\mathrm{O}}\left(r_{\|}\right)$ are also shown in Figure 5. For the three ethanol concentrations studied, the multiple pronounced peaks in the oxygen-oxygen $g_{\mathrm{O}-\mathrm{O}}\left(r_{\|}\right)$suggest that there is strong ordering in the 


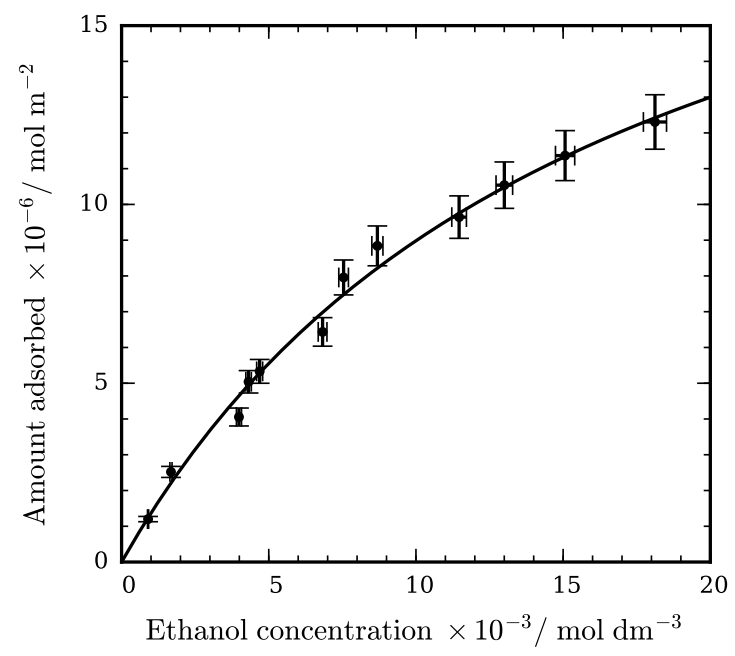

Figure 7: Adsorption isotherm of ethanol from iso-octane. Symbols represents data points and solid line represents fitted line.

first layer. The first peak of $g_{\mathrm{O}-\mathrm{O}}\left(r_{\|}\right)$is a result of the contact distance between two oxygen atoms, which for the OPLS force field correspond to $3.12 \AA$. The remaining peaks observed in the $g_{\mathrm{O}-\mathrm{O}}\left(r_{\|}\right)$coincide with the peaks observed in the iron-iron $g_{\mathrm{Fe}-\mathrm{Fe}}\left(r_{\|}\right)$. This suggests that the ordering of ethanol molecules follows closely the hexagonal arrangement of the iron atoms of the hematite surface. Regardless of the concentration of ethanol, the positions of the different peaks in the $g_{\mathrm{O}-\mathrm{O}}\left(r_{\|}\right)$are the same, although the intensity of the first peak varies slightly. The ordering of the oxygen atoms with respect to the iron atoms in the solid slab is also evident from the density maps shown in Figure 5 where a quasi-hexagonal order is observed. For the lowest concentration of ethanol analysed, which is not enough to form a complete monolayer, the density map shows a very striking clustering of ethanol, which is a further evidence of the strong hydrogen bonding network formed by these molecules at the surface. Hydrogen bonding analysis is carried out to confirm the coordination effect of ethanol in the first layer. Here, we consider a hydrogen bond to be formed when the distance between the donor oxygen and acceptor hydrogen is between $1.50 \AA$ and $2.10 \AA$. The analysis of the results reveal that there are $0.13,0.17$, and 0.23 hydrogen bonds per ethanol for the 

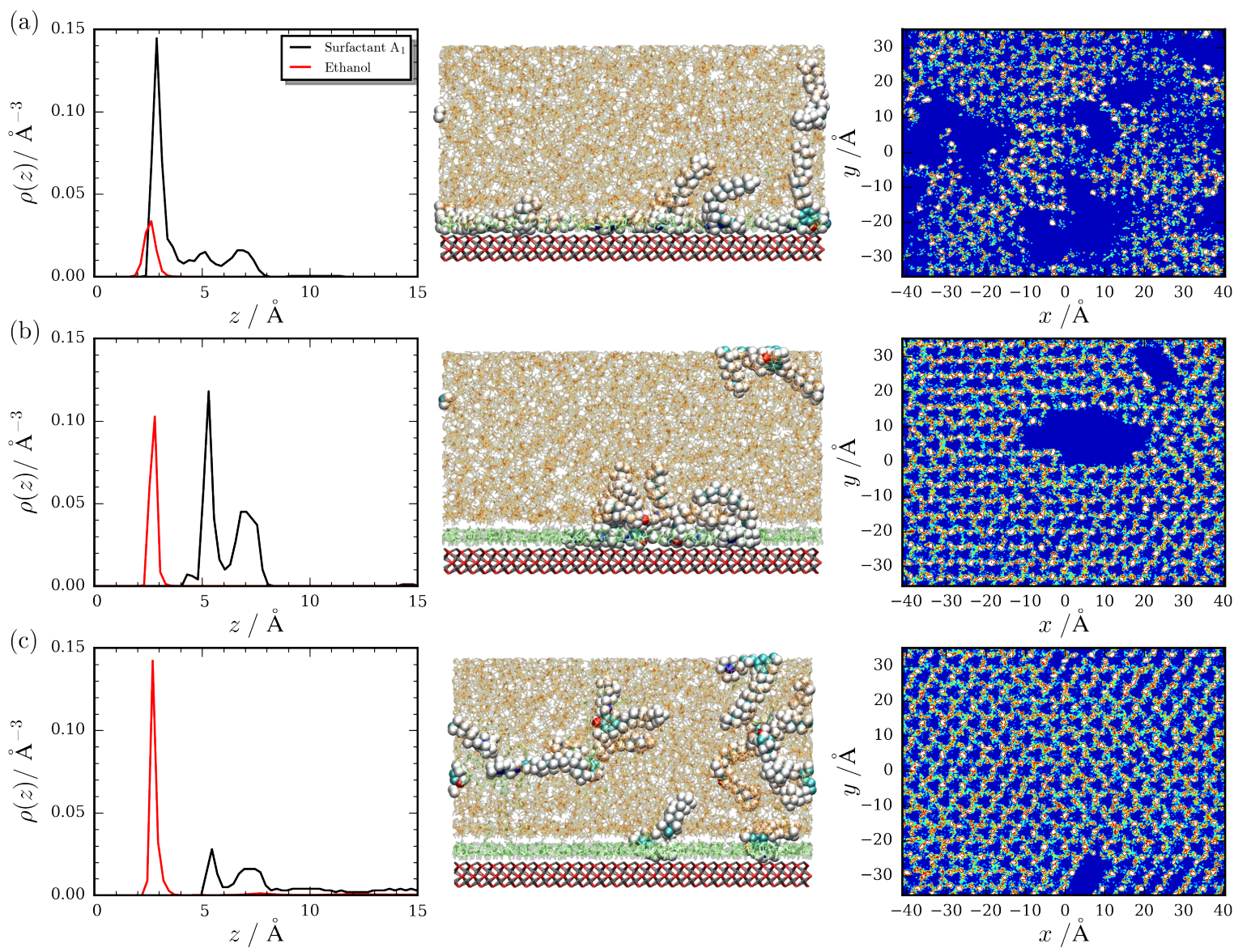

Figure 8: Density profiles $\rho(z)$ of surfactant $\mathrm{A}_{1}$ and ethanol along the normal direction to the surface for systems with 16 surfactant molecules, representative configuration for each ethanol concentration and two-dimensional density map of ethanol on hematite (0001) surface. The total number of ethanol molecules $N_{\text {ethanol }}$ in the three systems correspond to (a) 256, (b) 512 and (c) 768 molecules, respectively. The origin $z=0$ for the density profile corresponds to the position of the outermost solid atoms of the hematite slab. Note that the densities of surfactant $\mathrm{A}_{1}$ has been multiplied by 100 . Colour scheme for representation configuration: iso-octane (orange), ethanol (green) and surfactant $A_{1}$ (ball and stick model with CPK coloring).

three ethanol concentrations studied, respectively. From these results, it is evident that the hydrogen bond network also strengthens as the concentration of ethanol is increased.

We have also analysed the order of the ethanol layers as a function of the temperature. Calculations for the $g_{\mathrm{O}-\mathrm{O}}\left(r_{\|}\right)$for three temperatures corresponding to $293 \mathrm{~K}, 333 \mathrm{~K}$, and $373 \mathrm{~K}$, are carried out using a system comprising $N_{\text {ethanol }}=768$ ethanol molecules. The 
results, shown in Figure 6, reveal that the peaks observed in the $g_{\mathrm{O}-\mathrm{O}}(r)$ remain at the same positions for all temperatures explored but the intensities of the second peak decrease as the temperature is increased with values of $1.81,1.70$, and 1.65 , respectively. This indicates that the ordering in the first layer decreases slightly as the temperature increases, which is in agreement with the results shown previously in Figure 3(b) for the destabilisation of the ethanol layers with temperature according to the PMF calculations.

Based on the PMF analysis, ethanol molecules are expected to occupy the entire surface when there are more than $\sim 440$ ethanol molecules in the system (see Figure 4 ). Beyond this concentration, the adsorption of a single $\mathrm{A}_{1}$ molecule at $293 \mathrm{~K}$ should not be favourable due to the presence of a competitive species. With this in mind, we have carried out molecular-dynamics simulations of systems comprising $16 \mathrm{~A}_{1}$ molecules at three different ethanol concentrations, corresponding to 256, 512, and 768 ethanol molecules, respectively, at a temperature of $293 \mathrm{~K}$. For each simulation, 150 ns have been used for equilibration of the system, followed by a production run of $50 \mathrm{~ns}$. The results for the density profiles of $\mathrm{A}_{1}$ along the $z$ direction and representative configurations of the system are presented in Figure 8. For the system comprising 256 ethanol molecules (see Figure 8(a)), we observe that the first peaks of the density profile are close to the surface, which indicates that both ethanol and $\mathrm{A}_{1}$ are completely adsorbed at the oil/solid interface. This is expected, since at this low concentration of ethanol, the equilibrium between $A_{1}$ and ethanol shifts to a position where both can access the adsorption sites on the surface. From the density profile and from the snapshot at this low ethanol concentration, we can observe the surfactant molecules mainly adopt an adsorption conformation corresponding to Stage 1, i.e. the molecules of $\mathrm{A}_{1}$ adopt a flat conformation with respect to the surface. For the system comprising 512 molecules, few $A_{1}$ molecules are still able to adsorb by forming an aggregate at the oil/solid interface as inferred from the snapshot and the two-dimensional density map. This aggregation phenomenon is commonly observed in surfactant systems. ${ }^{15}$ However, the adsorption affinity of $A_{1}$ is reduced as inferred from the less intense first peak in the density profile shown in 


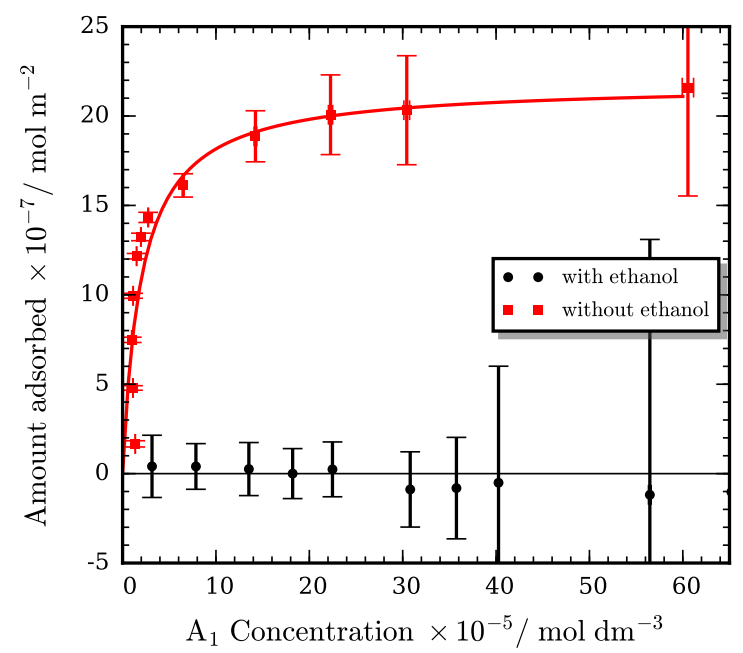

Figure 9: Adsorption isotherm of $\mathrm{A}_{1}$ with and without the presence of ethanol from isooctane. Symbols represents data points and solid line represents fitted line.

Figure $8(\mathrm{~b})$, and the development of a strong second peak. Finally, as the number of ethanol molecules is increased to 768 , the intensity of the two peaks in the density profile of $\mathrm{A}_{1}$ decreases significantly and most of the surfactant molecules remain in the oil phase, confirming the destabilisation in the adsorption of $A_{1}$ due to the saturation of the adsorption sites by the ethanol molecules.

The experimental results for the adsorption isotherm of $A_{1}$ onto the iron oxide from iso-octane are shown in Figure 9. The adsorption isotherm of $\mathrm{A}_{1}$ in the absence of ethanol shows a pronounced rise from zero as the total concentration of $\mathrm{A}_{1}$ increases until a plateau is reached. The data set has been fit with a Langmuir model allowing to estimate an area per molecule of $74.9 \pm 1.1 \AA^{2}$ and binding constant for adsorption $K$ of $48000 \pm 8000$, which indicates a fully covered surface with the $A_{1}$ molecules essentially upright. However, the addition of $5 \%$ ethanol by volume, which is much higher than the bulk concentrations used in simulations, completely changes the behaviour of the system. The ethanol concentration of $5 \%$ by volume is chosen as representative of a gasoline fuel composition. The results also shown in Figure 9 clearly indicates that the $A_{1}$ is displaced by ethanol thus confirming the 
results observed from the MD simulations. Note that negative values observed in Figure 9 reflect the statistical spread of the data -the magnitude of the measurement is small compared to the error.

\section{Conclusions}

In conclusion, we have demonstrated that the strong adsorption affinity of a model of a Mannich base surfactant, which contains two polar amino groups and a phenol group, on hematite is reduced considerably by the presence of ethanol. Using molecular-dynamics simulation we observed the destabilisation of the adsorption of $\mathrm{A}_{1}$ on hematite takes place when the (0001) surface of hematite is saturated with ethanol, and beyond this point the adsorption of $A_{1}$ is not longer stable and the surfactant molecules are transferred to the oil phase. Since ethanol is soluble in iso-octane at room temperature, the formation of the ethanol monolayer is entirely driven by the strong interaction of ethanol and the solid substrate as well as by the formation of a hydrogen bond network that increases the order of the monolayer as revealed by the radial distribution functions ( Figure 5) calculated with respect to the oxygen atoms of ethanol. As the temperature of the system is increased, the order in the ethanol monolayer decreases due to the destabilization of the hydrogen bond network that allows $A_{1}$ to compete effectively for the adsorption sites of hematite. These results are confirmed from our experimental results for the adsorption isotherms of $\mathrm{A}_{1}$ on hematite with and without ethanol. The simulation at high temperature, however, should not be taken for granted since it is known that, in real experiments, Mannich base surfactants are highly reactive on metal oxide surfaces and molecular simulations cannot describe completely the adsorption behaviour. For this case, simulations using reactive force fields such as ReaxFF ${ }^{56}$ should be able to provide a better insight into the competitive adsorption of ethanol and high-performance surfactants at high temperature and high pressure conditions. This will be the focus of future work. 


\section{Acknowledgements}

The authors would like to acknowledge the funding and technical support from BP through the BP International Centre for Advanced Materials (BP-ICAM), which made this research possible. CLC also acknowledges the support from the University of Manchester Alumni Scholarship. The authors would like to acknowledge the assistance given by IT Services and the use of the Computational Shared Facility (CSF) at The University of Manchester.

\section{Associated Content}

\section{Supporting Information}

OPLS-AA Force field parameters; analysis of structural properties of ethanol adsorbed on iron oxide at different temperatures; density profiles of ethanol adsorbed on hematite.

\section{References}

(1) Hiemenz, P. C.; Rajagopalan, R. Principles of Colloid and Surface Chemistry; CRC press, 1997.

(2) Zerda, T. W.; Yuan, X.; Moore, S. M. Effects of Fuel Additives on the Microstructure of Combustion Engine Deposits. Carbon 2001, 39, 1589-1597.

(3) Groysman, A. Corrosion in Systems for Storage and Transportation of Petroleum Products and Biofuels; Springer, 2014.

(4) Wood, M. H.; Clarke, S. M. Neutron Reflectometry for Studying Corrosion and Corrosion Inhibition. Metals 2017, 7, 304.

(5) Wood, M. H.; Casford, M. T.; Steitz, R.; Zarbakhsh, A.; Welbourn, R. J. L.; 
Clarke, S. M. Comparative Adsorption of Saturated and Unsaturated Fatty Acids at the Iron Oxide/Oil Interface. Langmuir 2016, 32, 534-540.

(6) Wood, M. H.; Welbourn, R. J. L.; Charlton, T.; Zarbakhsh, A.; Casford, M. T.; Clarke, S. M. Hexadecylamine Adsorption at the Iron Oxide-Oil Interface. Langmuir 2013, 29, 13735-13742.

(7) Zhang, R.; Somasundaran, P. Advances in Adsorption of Surfactants and their Mixtures at Solid/Solution Interfaces. Adv. Colloid Interface Sci. 2006, 123, 213-229.

(8) Messe, L.; Perdigon, A.; Clarke, S.; Inaba, A.; Arnold, T. Alkane/Alcohol Mixed Monolayers at the Solid/Liquid Interface. Langmuir 2005, 21, 5085-5093.

(9) Paria, S.; Khilar, K. A Review on Experimental Studies of Surfactant Adsorption at the Hydrophilic Solid-Water Interface. Adv. Colloid Interface Sci. 2004, 110, 75-95.

(10) Arnold, T.; Dong, C.; Thomas, R.; Castro, M.; Perdigon, A.; Clarke, S.; Inaba, A. The Crystalline Structures of the Odd Alkanes Pentane, Heptane, Nonane, Undecane, Tridecane and Pentadecane Monolayers Adsorbed on Graphite at Submonolayer Coverages and from the Liquid. Phys. Chem. Chem. Phys. 2002, 4, 3430-3435.

(11) Turner, S. F.; Clarke, S. M.; Rennie, A. R.; Thirtle, P. N.; Cooke, D. J.; Li, Z. X.; Thomas, R. K. Adsorption of Sodium Dodecyl Sulfate to a Polystyrene/Water Interface Studied by Neutron Reflection and Attenuated Total Reflection Infrared Spectroscopy. Langmuir 1999, 15, 1017-1023.

(12) Allen, M. P.; Tildesley, D. J. Computer Simulation of Liquids; Oxford University Press: Oxford, 1987.

(13) Frenkel, D.; Smit, B. Understanding Molecular Simulation: from Algorithms to Applications, 2nd ed.; Academic press, 2002. 
(14) Leach, A. Molecular Modelling: Principles and Applications; Pearson Education; Prentice Hall, 2001.

(15) Shinto, H.; Tsuji, S.; Miyahara, M.; Higashitani, K. Molecular Dynamics Simulations of Surfactant Aggregation on Hydrophilic Walls in Micellar Solutions. Langmuir 1999, $15,578-586$.

(16) Dominguez, H. Structural Transition of the Sodium Dodecyl Sulfate (SDS) Surfactant Induced by Changes in Surfactant Concentrations. J. Phys. Chem. B 2011, 115, 1242212428.

(17) Dominguez, H. Self-Aggregation of the SDS Surfactant at a Solid-Liquid Interface. J. Phys. Chem. B 2007, 111, 4054-4059.

(18) Sammalkorpi, M.; Panagiotopoulos, A. Z.; Haataja, M. Structure and Dynamics of Surfactant and Hydrocarbon Aggregates on Graphite: A Molecular Dynamics Simulation Study. J. Phys. Chem. B 2008, 112, 2915-2921.

(19) Srinivas, G.; Nielsen, S.; Moore, P.; Klein, M. Molecular Dynamics Simulations of Surfactant Self-Organization at a Solid-Liquid Interface. J. Am. Chem. Soc. 2006, $128,848-853$.

(20) Hu, X.; Li, Y.; Sun, H.; Song, X.; Li, Q.; Cao, X.; Li, Z. Effect of Divalent Cationic Ions on the Adsorption Behavior of Zwitterionic Surfactant at Silica/Solution Interface. J. Phys. Chem. B 2010, 114, 8910-8916.

(21) Dominguez, H. Structure of the Sodium Dodecyl Sulfate Surfactant on a Solid Surface in Different $\mathrm{NaCl}$ Solutions. Langmuir 2009, 25, 9006-9011.

(22) Shah, K.; Chiu, P.; Jain, M.; Fortes, J.; Moudgil, B.; Sinnott, S. Morphology and Mechanical Properties of Surfactant Aggregates at Water-Silica Interfaces: Molecular Dynamics Simulations. Langmuir 2005, 21, 5337-5342. 
(23) Striolo, A.; Grady, B. P. Surfactant Assemblies on Selected Nanostructured Surfaces: Evidence, Driving Forces, and Applications. Langmuir 2017, 33, 8099-8113.

(24) Suttipong, M.; Grady, B. P.; Striolo, A. Self-assembled Surfactants on Patterned Surfaces: Confinement and Cooperative Effects on Aggregate Morphology. Phys. Chem. Chem. Phys. 2014, 16, 16388-16398.

(25) Farrow, M. R.; Camp, P. J.; Dowding, P. J.; Lewtas, K. The Effects of Surface Curvature on the Adsorption of Surfactants at the Solid-Liquid Interface. Phys. Chem. Chem. Phys. 2013, 15, 11653-11660.

(26) Nuñez Rojas, E.; Dominguez, H. Aggregate Structures of the Sorbitan Monooleate (SPAN80) Surfactant at TiO2(rutile)/Water Interfaces by Computer Simulations. Rev. Mex. Fis. 2013, 59, 530-539.

(27) Nuñez Rojas, E.; Dominguez, H. Computational Studies on the Behavior of Sodium Dodecyl Sulfate (SDS) at TiO2(rutile)/Water Interfaces. J. Colloid Interface Sci. 2011, $364,417-427$.

(28) Liu, Z.; Yu, J.-G.; O’Rear, E. A.; Striolo, A. Aqueous Dual-Tailed Surfactants Simulated on the Alumina Surface. J. Phys. Chem. B 2014, 118, 9695-9707.

(29) Zehl, T.; Wahab, A.; Schiller, P.; Moegel, H. J. Monte Carlo Simulation of Surfactant Adsorption on Hydrophilic Surfaces. Langmuir 2009, 25, 2090-2100.

(30) Zheng, F.; Zhang, X.; Wang, W.; Dong, W. Adsorption and Morphology Transition of Surfactants on Hydrophobic Surfaces: A Lattice Monte Carlo Study. Langmuir 2006, 22, 11214-11223.

(31) Doig, M.; Camp, P. J. The Structures of Hexadecylamine Films Adsorbed on IronOxide Surfaces in Dodecane and Hexadecane. Phys. Chem. Chem. Phys. 2015, 17, $5248-5255$. 
(32) Bradley-Shaw, J. L.; Camp, P. J.; Dowding, P. J.; Lewtas, K. Molecular Dynamics Simulations of Glycerol Monooleate Confined between Mica Surfaces. Langmuir 2016, 32, 7707-7718.

(33) Cornejo, A.; Barrio, I.; Campoy, M.; Lazaro, J.; Navarrete, B. Oxygenated Fuel Additives from Glycerol Valorization. Main Production Pathways and Effects on Fuel Properties and Engine Performance: A Critical Review. Renew. Sust. Energ. Rev. 2017, 79, $1400-1413$.

(34) Giakoumis, E. G.; Rakopoulos, C. D.; Dimaratos, A. M.; Rakopoulos, D. C. Exhaust Emissions with Ethanol or n-Butanol Diesel Fuel Blends during Transient Operation: A Review. Renew. Sust. Energ. Rev. 2013, 17, 170-190.

(35) Ribeiro, N. M.; Pinto, A. C.; Quintella, C. M.; da Rocha, G. O.; Teixeira, L. S. G.; Guarieiro, L. L. N.; do Carmo Rangel, M.; Veloso, M. C. C.; Rezende, M. J. C.; da Cruz, R. S. et al. The Role of Additives for Diesel and Diesel Blended (Ethanol or Biodiesel) Fuels: A Review. Energy Fuels 2007, 21, 2433-2445.

(36) Hansen, A. C.; Zhang, Q.; Lyne, P. W. L. Ethanol-Diesel Fuel Blends - A Review. Bioresour. Technol. 2005, 96, 277-285.

(37) Malfer, D.; Kadkhodayan, A.; Thomas, M. Fuel Additive Compounds and Method of Making the Compounds. 2008; US Patent App. 11/465,278.

(38) Ghosh, P.; Hoque, M. Mannich Bases and Phosphosulphurized Mannich Bases: Synthesis, Characterization and Performance Evaluation as Potential Lube Oil Additives. J. Sci. Ind. Res. 2015, 74, 150-154.

(39) Kamal, R. S.; Ahmed, N. S.; Nasser, A. M. Study the Efficiency of some Compounds as Lubricating Oil Additives. Appl. Petrochem. Res. 2013, 3, 1-8. 
(40) Maeda, S. Steel Surface Chemistry Affecting the Performance of Organic Coatings. Prog. Org. Coat. 1983, 11, 1-18.

(41) Sewell, P. B.; Stockbridge, C. D.; Cohen, M. An Electrometric and Electron Diffraction Study of Air-Formed Oxide Films on Iron. J. Electrochem. Soc. 1962, 109, 547-549.

(42) Dzade, N. Y.; Roldan, A.; de Leeuw, N. H. A Density Functional Theory Study of the Adsorption of Benzene on Hematite $\left(\alpha-\mathrm{Fe}_{2} \mathrm{O}_{3}\right)$ Surfaces. Minerals 2014, 4, 89-115.

(43) Finger, L. W.; Hazen, R. M. Crystal Structure and Isothermal Compression of $\mathrm{Fe}_{2} \mathrm{O}_{3}$, $\mathrm{Cr}_{2} \mathrm{O}_{3}$, and $\mathrm{V}_{2} \mathrm{O}_{3}$ to 50 kbars. J. Appl. Phys. 1980, 51, 5362-5367.

(44) Berro, H.; Fillot, N.; Vergne, P. Molecular Dynamics Simulation of Surface Energy and ZDDP Effects on Friction in Nano-Scale Lubricated Contacts. Tribol. Int. 2010, 43, $1811-1822$.

(45) Doig, M.; Warrens, C. P.; Camp, P. J. Structure and Friction of Stearic Acid and Oleic Acid Films Adsorbed on Iron Oxide Surfaces in Squalane. Langmuir 2014, 30, 186-195.

(46) Price, M. L. P.; Ostrovsky, D.; Jorgensen, W. L. Gas-Phase and Liquid-State Properties of Esters, Nitriles, and Nitro Compounds with the OPLS-AA Force Field. J. Comput. Chem. 2001, 22, 1340-1352.

(47) Sweere, A. J. M.; Fraaije, J. G. E. M. Accuracy Test of the OPLS-AA Force Field for Calculating Free Energies of Mixing and Comparison with PAC-MAC. J. Chem. Theory Comput. 2017, 13, 1911-1923.

(48) Outcalt, S. L.; Laesecke, A. Compressed-Liquid Densities of Two Highly Polar Plus Non-Polar Binary Systems. J. Mol. Liq. 2012, 173, 91-102.

(49) Plimpton, S. Fast Parallel Algorithms for Short-Range Molecular-Dynamics. J. Comput. Phys. 1995, 117, 1-19. 
(50) Martinez, L.; Andrade, R.; Birgin, E. G.; Martinez, J. M. PACKMOL: A Package for Building Initial Configurations for Molecular Dynamics Simulations. J. Comput. Chem. 2009, 30, 2157-2164.

(51) Nosé, S. A Unified Formulation of the Constant Temperature Molecular Dynamics Methods. J. Chem. Phys. 1984, 81, 511-519.

(52) Hoover, W. G. Canonical Dynamics - Equilibrium Phase-Space Distributions. Phys. Rev. A 1985, 31, 1695-1697.

(53) Kumar, S.; Bouzida, D.; Swendsen, R. H.; Kollman, P. A.; Rosenberg, J. M. The Weighted Histogram Analysis Method for Free-Energy Calculations on Biomolecules. 1. The Method. J. Comput. Chem. 1992, 13, 1011-1021.

(54) Lide, D. R. In CRC Handbook of Chemistry and Physics, 98th ed.; Rumble, J. R., Ed.; CRC Press, 2017; p 529.

(55) Phan, A.; Cole, D. R.; Striolo, A. Liquid Ethanol Simulated on Crystalline Alpha Alumina. J. Phys. Chem. B 2013, 117, 3829-3840.

(56) van Duin, A. C. T.; Dasgupta, S.; Lorant, F.; Goddard, W. A. ReaxFF: A Reactive Force Field for Hydrocarbons. J. Phys. Chem. A 2001, 105, 9396-9409. 


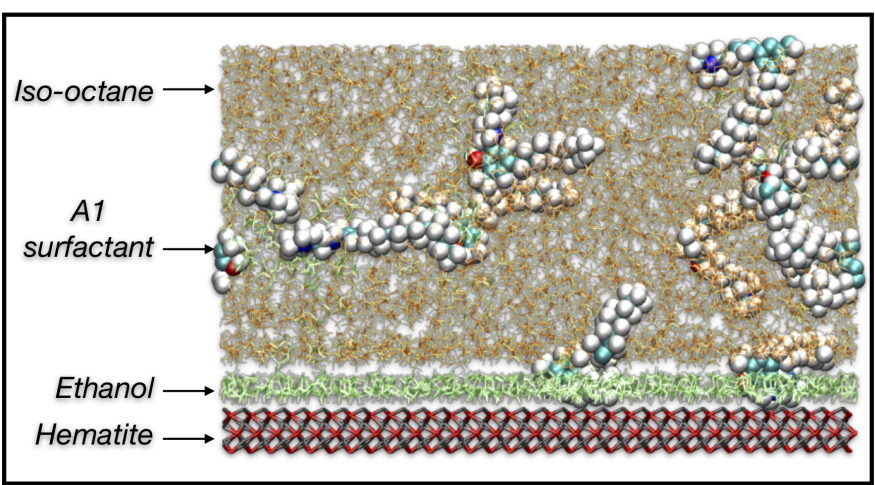

